\title{
RESEARCH
}

Open Access

\section{Plasma biomarker profiles and the correlation with cognitive function across the clinical spectrum of Alzheimer's disease}

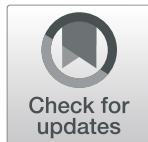

Zhenxu Xiao ${ }^{1,2+}$, Xue $\mathrm{Wu}^{3+}$, Wanqing $\mathrm{Wu}^{1,2}$, Jingwei $\mathrm{Yi}^{3}$, Xiaoniu Liang ${ }^{1,2}$, Saineng Ding ${ }^{1,2}$, Li Zheng ${ }^{1,2}$, Jianfeng Luo ${ }^{4,5}$, Hongchen $\mathrm{Gu}^{3}$, Qianhua Zhao ${ }^{1,2^{*}}$, Hong $\mathrm{Xu}^{3^{*}}$ and Ding Ding ${ }^{1,2}$

\begin{abstract}
Background: Plasma biomarkers showed a promising value in the disease diagnosis and management of Alzheimer's disease (AD). However, profiles of the biomarkers and the associations with cognition across a spectrum of cognitive stages have seldom been reported.

Methods: We recruited 320 individuals with cognitive impairment and 131 cognitively normal participants from a memory clinic and a community cohort. Participants were classified into 6 groups based on their Clinical Dementia Rating (CDR) scores and clinical diagnosis, including $A D$, amnestic mild cognitive impairment ( $\mathrm{aMCl}$ ), and normal cognition (NC). A battery of neuropsychological tests was used to assess the global and domain-specific cognition. Plasma $A \beta_{1-40}, A \beta_{1-42}, A \beta_{1-42} / A \beta_{1-40}$, total tau (t-tau), neurofilament protein light chain (NfL), and phosphorylated tau at threonine 181 (p-tau181) were quantified using the single-molecule array (Simoa) platform.

Results: All the plasma markers ( $A \beta_{1-40}, A \beta_{1-42}, A \beta_{1-42} / A \beta_{1-40}, t$-tau, NfL, $p$-tau181) showed certain discrepancies among $\mathrm{NC}$, aMCl, and AD groups. The p-tau181 level showed a continuous escalating trend as the CDR scores increased from 0 (NC group) to 3 (severe AD). Compared with other biomarkers, p-tau181 had correlations with broader cognitive domains, covering global cognition $(r=-0.536, P<0.0001)$, memory $(r=-0.481, P<0.0001)$, attention $(r=-0.437, P<0.0001)$, visuospatial function $(r=-0.385, P<0.0001)$, and language $(r=-0.177, P=0.0003)$. Among participants with $C D R \geq 1$, higher $p$-tau181 was correlated with worse global cognition $(r=-0.301, P<0.001)$.
\end{abstract}

Conclusions: Plasma p-tau181 had correlations with broader cognitive domains, suggesting its potential as a promising clinical-relevant blood-based biomarker.

Keywords: Plasma p-tau181, Simoa, Cognitive domain, CDR, Alzheimer's disease

\footnotetext{
*Correspondence: qianhuazhao@fudan.edu.cn; xuhong@sjtu.edu.cn

${ }^{\dagger}$ Zhenxu Xiao and Xue Wu contributed equally to this work.

'Institute of Neurology, Huashan Hospital, Fudan University, 12 Wulumuqi

Zhong Rd, Shanghai, China

${ }^{3}$ School of Biomedical Engineering, Shanghai Jiao Tong University, Shanghai,

China

Full list of author information is available at the end of the article
}

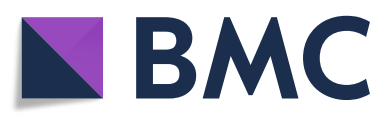

(- The Author(s). 2021 Open Access This article is licensed under a Creative Commons Attribution 4.0 International License, which permits use, sharing, adaptation, distribution and reproduction in any medium or format, as long as you give appropriate credit to the original author(s) and the source, provide a link to the Creative Commons licence, and indicate if changes were made. The images or other third party material in this article are included in the article's Creative Commons licence, unless indicated otherwise in a credit line to the material. If material is not included in the article's Creative Commons licence and your intended use is not permitted by statutory regulation or exceeds the permitted use, you will need to obtain permission directly from the copyright holder. To view a copy of this licence, visit http://creativecommons.org/licenses/by/4.0/. The Creative Commons Public Domain Dedication waiver (http://creativecommons.org/publicdomain/zero/1.0/) applies to the data made available in this article, unless otherwise stated in a credit line to the data. 


\section{Background}

Alzheimer's disease (AD), the most common cause of dementia, is characterized by the accumulation of the amyloid plaques and neuronal tangles in the brain [1]. Previous AD-relevant biomarkers could only be detected in the cerebrospinal fluid (CSF) or through positron emission tomography (PET) [1]. With the development of ultrasensitive immunoassays technique, detecting $\mathrm{AD}$ relevant biomarkers in blood samples became available [2]. Plasma biomarkers have a promising value in clinic usage due to the non-invasiveness, cost-effectiveness, and easy accessibility [3]. Following $A \beta_{1-42}, A \beta_{1-40}$, total tau ( $\mathrm{t}$-tau), neurofilament protein light chain (NfL), recently reported plasma phosphorylated tau at threonine 181 (p-tau181) showed better diagnostic performance and prognostic value in several cohort studies [3-9].

The cognitive performance is a pivotal indicator in AD management and efficacy evaluation. Previous studies found plasma $A \beta_{1-42}[10], \mathrm{NfL}[11,12]$, and p-tau181 $[3,4,13]$ were significantly different in participants with mild cognitive impairment (MCI) and AD compared with participants with normal cognition (NC). However, few studies depicted plasma biomarkers' profiles based on the cognitive performance and compared their discrepancy in different stages of AD clinical syndrome. It would be valuable to reveal the alterations of plasma AD biomarkers from NC until the severe cognitive impairment stage since the cognitive manifestations are the most concerned issues for both clinicians, patients, and caregivers.

In addition, some studies investigated the relationships between plasma biomarkers and various cognitive domains [11, 14-22]. However, they only focused on individual markers, and the results were incomparable or inconsistent due to the diverse testing platforms and study designs. It is also worthwhile to observe which biomarker has the best correlation with the cognitive domains.

In this study, we aimed to depict the profile of plasma biomarkers, including $A \beta_{1-40}, A \beta_{1-42}, A \beta_{1-42} / A \beta_{1-40}$, ttau, NfL, and p-tau181, among different stages of $A D$ clinical syndrome. We also intended to explore the correlation between these biomarkers and the domainspecific cognitive functions.

\section{Methods}

\section{Study participants}

Participants with cognitive impairment were consecutively recruited from the memory clinic of the department of neurology, Huashan Hospital, Shanghai, China from November 2018 to September 2020. The inclusion criteria included the following: (1) consulted at the memory clinic due to memory complaints from herself (himself) or proxy; (2) able to cooperate with physical examinations and neuropsychological tests; (3) diagnosed with single-domain amnestic MCI (aMCI-s), multi-domain amnestic MCI (aMCI-m), or AD clinical syndrome; and (4) consent to the blood draw.

Participants in the Shanghai Aging Study (SAS) were eligible to be selected as the controls with NC. The SAS is a community-based longitudinal cohort in downtown Shanghai, China. The original purpose of SAS was to investigate the prevalence, incidence, and risk factors for dementia and MCI among older residents in an urban community. The detailed study design and recruitment procedure have been published elsewhere [23]. In this study, the participants were selected from the third wave of follow-up between Jun and Oct in 2020 if they were (1) 60 years or older, (2) able to cooperate with physical examinations and neuropsychological tests, (3) with normal cognition, and (4) consent to the blood draw.

\section{Demographics and assessment of covariables}

The demographic and lifestyle characteristics were acquired from the participants and/or proxy through a questionnaire. The educational background was defined as the years of formal education. Participants who smoked daily within the past month were regarded as cigarette smoking, and alcohol consumption was defined as having at least one serving of alcohol weekly during the past year [24]. Hypertension and diabetes mellitus were confirmed by the medical records [23].

The Barthel index [25] and the Brody Activity of Daily Living (ADL) scale-16 [26] were used to elicit physical self-maintenance and instrumental activities of daily living, such as eating, preparing meals, using the telephone, handling money, and doing chores, for participants from the clinic and the community, respectively. Participants were considered functionally intact if the Barthel index score was over 60 [25], or the ADL score was over 16 [26]. Apolipoprotein E (APOE) genotype was assessed by the Taqman single-nucleotide polymorphism method using the blood or saliva samples collected from the participants [27]. The presence of at least one APOE $\varepsilon 4$ allele was regarded as APOE \&4-positive.

\section{Neuropsychological tests}

Comprehensive neuropsychological tests were administered by the certified psychometrists. All the tests were translated and adapted from western countries harmonized to Chinese culture and were validated in the Chinese population. Each participant from the memory clinic received a battery of neuropsychological tests, including (1) mini-mental status examination (MMSE) [28], (2) Montreal Cognitive Assessment-Basic (MoCAB) [29-31], (3) Auditory Verbal Learning Test [32, 33], (4) Symbol Digit Modalities Test [34, 35], (5) ReyOsterrieth Complex Figure test [35], (6) Boston Naming Test [36], and (7) Trail Making Test (TMT) [37]. For 
those who refused or were unable to complete the whole battery of tests, only MMSE and MoCA-B were administered. Because the MMSE is less sensitive for MCI detection [38], MoCA was used together with MMSE to discriminate dementia and MCI. As for the participants with NC, a battery of similar neuropsychological tests was administered due to the original study design of SAS [32]: (1) MMSE [28], (2) Auditory Verbal Learning Test [32, 33], (3) Conflicting Instructions Task (Go/NoGo Task) [32], (4) Stick Test [32], (5) Modified Common Objects Sorting Test [32], and (6) TMT [37].

In order to make the best use of the neuropsychological tests, we extracted raw scores from each test to evaluate five clinically relevant cognitive domains including Memory, Attention, Visuospatial function, Language, and Executive function (Supplementary Table 1). The percentage of the correct answer in each domain was calculated. Then, $Z$ scores were further computerized to ensure the comparability between the participants from the two cohorts.

\section{Cognitive impairment severity and consensus diagnosis}

All participants and their proxy were interviewed by two neurologists specialized in neurodegenerative diseases. The CDR, a semi-structured inventory, was used to assess the severity of cognitive impairment. It covered six cognitive, behavioral, and functional aspects, including memory, orientation, judgment and problem-solving, community affairs, home and hobby performance, and personal care. The neurologists scored on each aspect, taking into consideration information collected from both participants and proxy. The global CDR score was calculated automatically on biostat.wustl.edu/ adrc/ cdrpgm/index.html by inputting in each CDR box score, based on the Washington University CDR-assignment algorithm with a $0-3$ scale index $[39,40]$.

Neurologists and neuropsychologists reached a consensus diagnosis of the cognitive function of each participant. The presence or absence of dementia was defined using the DSM-IV criteria [41]: (1) memory impairment; (2) at least one of the following cognitive impairments: aphasia, apraxia, agnosia, and executive dysfunction; (3) cognitive deficit results in significant impairment of social or professional functioning; (4) cognitive function declines slowly; and (5) exclude other potential disorders that contribute to cognitive decline. Probable AD was diagnosed according to the NINCDSADRDA criteria [42]: (1) dementia established by MMSE and confirmed by neuropsychological tests, (2) deficits in two or more areas of cognition, (3) progressive worsening of memory and other cognitive functions, (4) no disturbance of consciousness, and (5) the laboratory tests (including, thyroid function, syphilis tests, level of vitamin B12 and folate, and other related blood tests) and magnetic resonance imaging (MRI)/computerized tomography $(\mathrm{CT})$ was performed to rule out other systemic disorders or other brain diseases that would cause cognitive decline. Participants who met the criterion of probable $\mathrm{AD}$ were regarded as having $\mathrm{AD}$ clinical syndrome. The diagnosis of MCI was based on Petersen's criteria [43]: (1) cognitive complaint by the subject, informant, nurse, or physician, with $C D R=0.5$; (2) objective impairment in at least 1 cognitive domain; (3) essentially normal functional activities (determined from the CDR and the ADL score); (4) absence of dementia; and (5) the laboratory tests and MRI/CT was performed to rule out other systemic disorders or other brain diseases that would cause cognitive decline [44]. Because aMCI is more likely to progress to $\mathrm{AD}$ [45], we only included participants with 2 types of aMCI: (1) aMCI-s, memory impairment was required with no deficit in other domains, and (2) aMCI-m, memory impairment plus at least 1 additional deficit in another domain. NC participants had no memory complaint and have been confirmed cognitively intact through detailed neuropsychological assessment.

In this study, the continuum of AD clinical syndrome was described based on the combination of CDR and clinical diagnosis: $\mathrm{NC}(\mathrm{CDR}=0)$, aMCI-s $(\mathrm{CDR}=0.5)$, aMCI-m $(\mathrm{CDR}=0.5)$, mild $\mathrm{AD}(\mathrm{CDR}=1)$, moderate $\mathrm{AD}(\mathrm{CDR}=2)$, and severe $\mathrm{AD}(\mathrm{CDR}=3)[39,40]$.

\section{Plasma biomarker measurement}

Blood samples were collected in ethylene diamine tetraacetic acid (EDTA) plasma tubes and centrifuged (1000 $\mathrm{rpm}, 4^{\circ} \mathrm{C}$ ) for $15 \mathrm{~min}$. After the centrifugation, plasma was transferred into $1.5 \mathrm{ml}$ Eppendorf tubes and stored at $-80^{\circ} \mathrm{C}$ refrigerators.

Plasma $A \beta_{1-40}, A \beta_{1-42}, t$-tau, NfL, and $p$-tau181 were quantified using an ultra-sensitive single-molecule array (Simoa) (Quanterix, MA, USA) on the automated Simoa HD-X platform per the manufacturer's instruction. The multiplex Neurology 3-Plex A kits (Cat. No. 101995), NF-light assay (Cat. No. 103186), and p-tau181 Assay Kit V2 (Cat. No.103714) were purchased from Quanterix and used accordingly. Technicians who performed the assay were blinded to the clinical data.

\section{Statistical analyses}

Mean and standard deviation (SD) were used to describe normally distributed continuous variables, while the median and quartile $1(\mathrm{Q} 1)$ to quartile $3(\mathrm{Q} 3)$ were used to describe the skewed distributed continuous variables. For categorical variables, number (n) and frequencies (\%) were employed. One-way analyses of variance (ANOVA) and Kruskal-Wallis tests were used for comparing continuous variables, and Pearson chi-square and Fisher exact tests were used to compare the categorical variables. For the comparisons among multi-groups, 
ANOVA and post hoc Tukey multiple comparison tests were used for variables with equal variance, while Welch and post hoc Games-Howell tests were used for variables with unequal variance.

Boxplots and points were used to present the distributions of original values of six plasma biomarkers. Levene's tests were used for testing the homogeneity of variances in six groups with different cognition statuses. Associations between domain-specific $Z$ scores and $\log$ transformed plasma biomarker indexes (due to nonnormal distributions [3]) were examined using partial correlation analyses with the adjustment for age, gender, and education year. The heatmap matrix was implemented to visualize the adjusted partial correlation coefficients $r$ in all participants. Positive correlations $(r>0)$ were exhibited in red, and negative correlations $(r<0)$ were exhibited in blue in the heatmap figure. The same correlation analyses were applied to participants with $\mathrm{CDR}=0, \mathrm{CDR}=0.5$, and $\mathrm{CDR} \geq 1$, respectively. For partial correlation analysis, we used the Bonferroni method for multiple corrections [46]. Since global cognition and memory are the two most important cognition domains for $\mathrm{AD}$ clinical syndrome, scatter plots were used to illustrate the correlations between MMSE \& memory and plasma biomarkers.

Two-sided $P<0.05$ was considered statistically significant, except for a specific explanation of multiple corrections. The degree of freedoms (df) was presented in correlation analysis. Data were analyzed using IBM SPSS Statistics for Windows, version 25.0 (IBMCorp., Armonk, N.Y., USA) [47] and R (version 4.0.2) [48]. Box plots were produced using the package ggplot2 in R. The heatmap was visualized by GraphPad Prism version 8.0.0 for Windows, GraphPad Software, San Diego, California, USA (www.graphpad.com).

\section{Results}

\section{Characteristics of the study participants}

We recruited 451 participants, including 320 participants from the memory clinic cohort and $131 \mathrm{NC}$ participants from the community cohort. The characteristics of study participants were shown in Table 1. Significant difference was found in gender $(P=0.011)$, age $(P<0.001)$, education years $(P<0.001)$, and APOE $\varepsilon 4$ allele $(P<$ 0.001 ) among six cognitive performance groups. The most severe cognitive impairment group $(\mathrm{CDR}=3)$ had the highest proportion of women $(68.4 \%)$, the lowest mean age $($ mean $=62.3)$, the shortest education years $($ mean $=5.6)$, and the largest proportion of positive APOE $\varepsilon 4$ allele $(57.9 \%)$. We did not find a significant discrepancy in smoking, alcohol consumption, hypertension, and diabetes mellitus among the six groups. All neuropsychological test scores were significantly different among six groups (all $P<0.001$ ).
Plasma biomarkers across groups with different cognitive performances

As shown in Fig. 1, plasma $A \beta_{1-40}, A \beta_{1-42}$, and $A \beta_{1-42} /$ $\mathrm{A} \beta_{1-40}$ ratio showed a descending trend, while plasma ttau, NfL, and p-tau181 exhibited an increasing trend across groups with the increasing CDR scores in general. With regard to $A \beta_{1-40}, A \beta_{1-42}$, and NfL, we found significant differences between participants with $\mathrm{NC}(\mathrm{CDR}=$ $0)$ and $\mathrm{AD}(\mathrm{CDR} \geq 1)$ (Fig. $1 \mathrm{~A}, \mathrm{~B}$, and $\mathrm{E}) . \mathrm{A} \beta_{1-42} / \mathrm{A} \beta_{1-40}$ and $t$-tau showed differences only between participants with $\mathrm{NC}(\mathrm{CDR}=0)$ and severe $\mathrm{AD}(\mathrm{CDR}=3)($ Fig. $1 \mathrm{C}$ and $D)$. There was no significant discrepancy of $A \beta_{1-40}$, $A \beta_{1-42}, A \beta_{1-42} / A \beta_{1-40}, t$-tau, or NfL among participants with different severity levels of $\mathrm{AD}(\mathrm{CDR}=1,2$, or 3$)$. P-tau181 gradually increased among different cognitive stages of AD clinical syndrome, with the lowest concentration in NC participants $(\mathrm{CDR}=0)$, an increase in participants with aMCI $(C D R=0.5)$, and the highest concentration in participants with $\mathrm{AD}(\mathrm{CDR} \geq 1)$ (Fig. 1F). Specifically, participants with severe AD $(\mathrm{CDR}=3)$ had significantly higher $\mathrm{p}$-tau181 than those with mild AD (CDR =1).

When we compared the difference of plasma biomarkers between $\mathrm{CDR}=0.5$ and $\mathrm{CDR}>=1$, it turned out that plasma $\mathrm{A} \beta_{1-42}(P=0.025), \mathrm{t}$-tau $(P=0.010)$, and $\mathrm{p}$-tau181 $(P<0.001)$ showed significant difference (not shown in Fig. 1).

\section{Correlation between plasma biomarkers and domain- specific cognition}

Figure 2 showed the partial correlation matrix between six plasma biomarkers and six domain-specific cognitions after adjusting age, gender, and education years. Through Bonferroni correction, $A \beta_{1-42}$ showed a positive correlation with MMSE $(r=0.156, \mathrm{df}=444, P=0.0010)$ and memory $(r=0.244, \mathrm{df}=408, P<0.0011) . \mathrm{A} \beta_{1-42} / \mathrm{A} \beta_{1-40}$ was only positively correlated with memory $(r=0.161, \mathrm{df}=407, P=$ 0.0011). T-tau had an inverse correlation with MMSE ( $r=$ $-0.168, \mathrm{df}=444, P=0.0004)$. Higher NFL was correlated with worse MMSE $(r=-0.322, \mathrm{df}=441, P<0.0001)$, memory $(r=-0.292, \mathrm{df}=405, P<0.0001)$, attention $(r=-0.236$, $\mathrm{df}=406, P<0.0001)$, and visuospatial function $(r=-0.264$, $\mathrm{df}=378, P<0.0001)$. P-tau181 showed a stronger negative correlation with MMSE $(r=-0.536, \mathrm{df}=441, P<0.0001)$, memory $(r=-0.481, \mathrm{df}=406, P<0.0001)$, attention $(r=$ -0.437, $\mathrm{df}=407, P<0.0001)$, visuospatial function $(r=$ $-0.385, \mathrm{df}=379, P<0.0001)$, and language $(r=-0.177, \mathrm{df}$ $=406, P=0.0003)$. Figures 3 and 4 demonstrated the scatter plots of the correlations between MMSE \& memory and six plasma biomarkers, respectively.

When the participants were divided into three stages according to the CDR score, only higher p-tau181 was correlated with worse MMSE in participants with CDR 


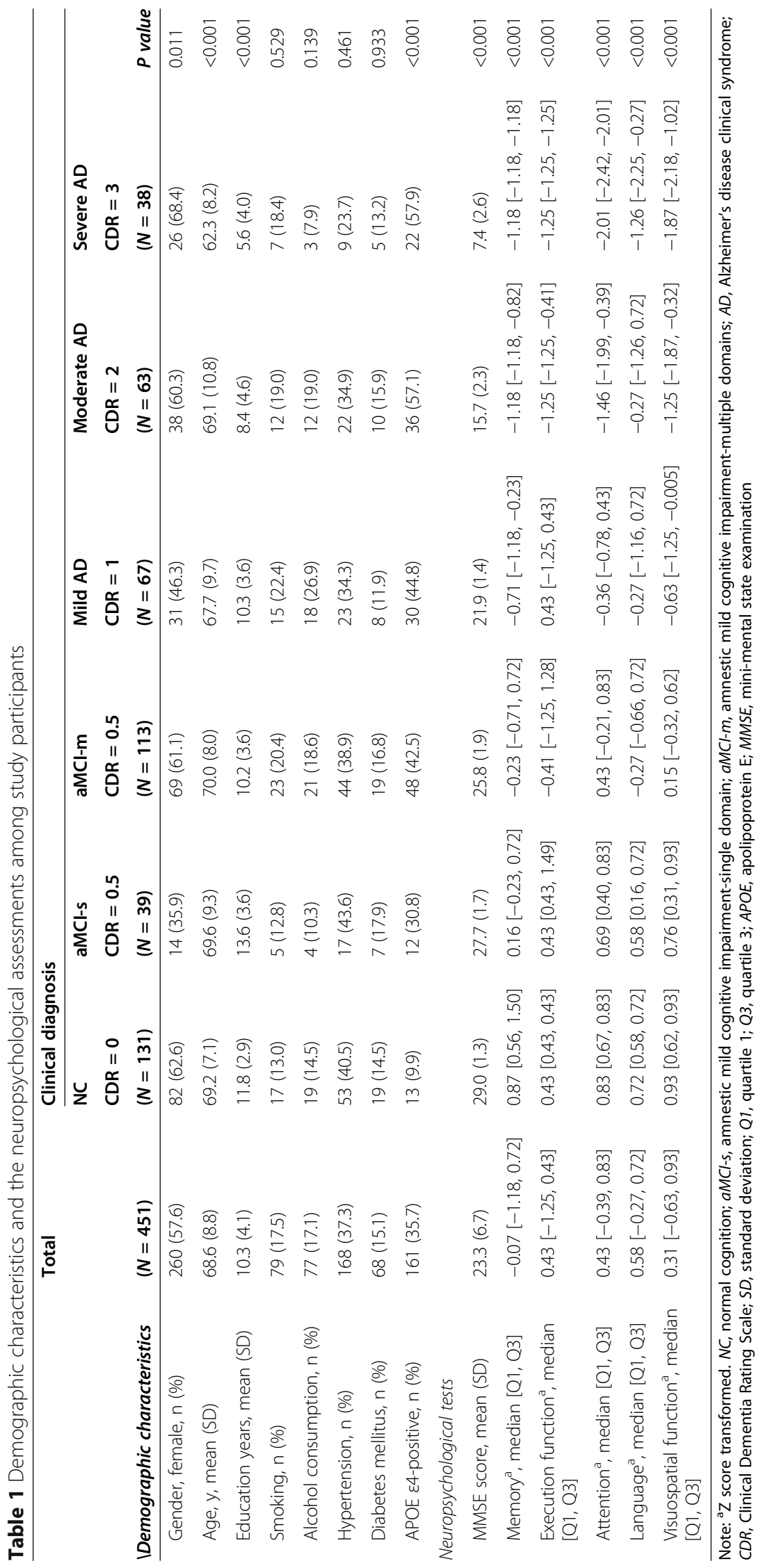



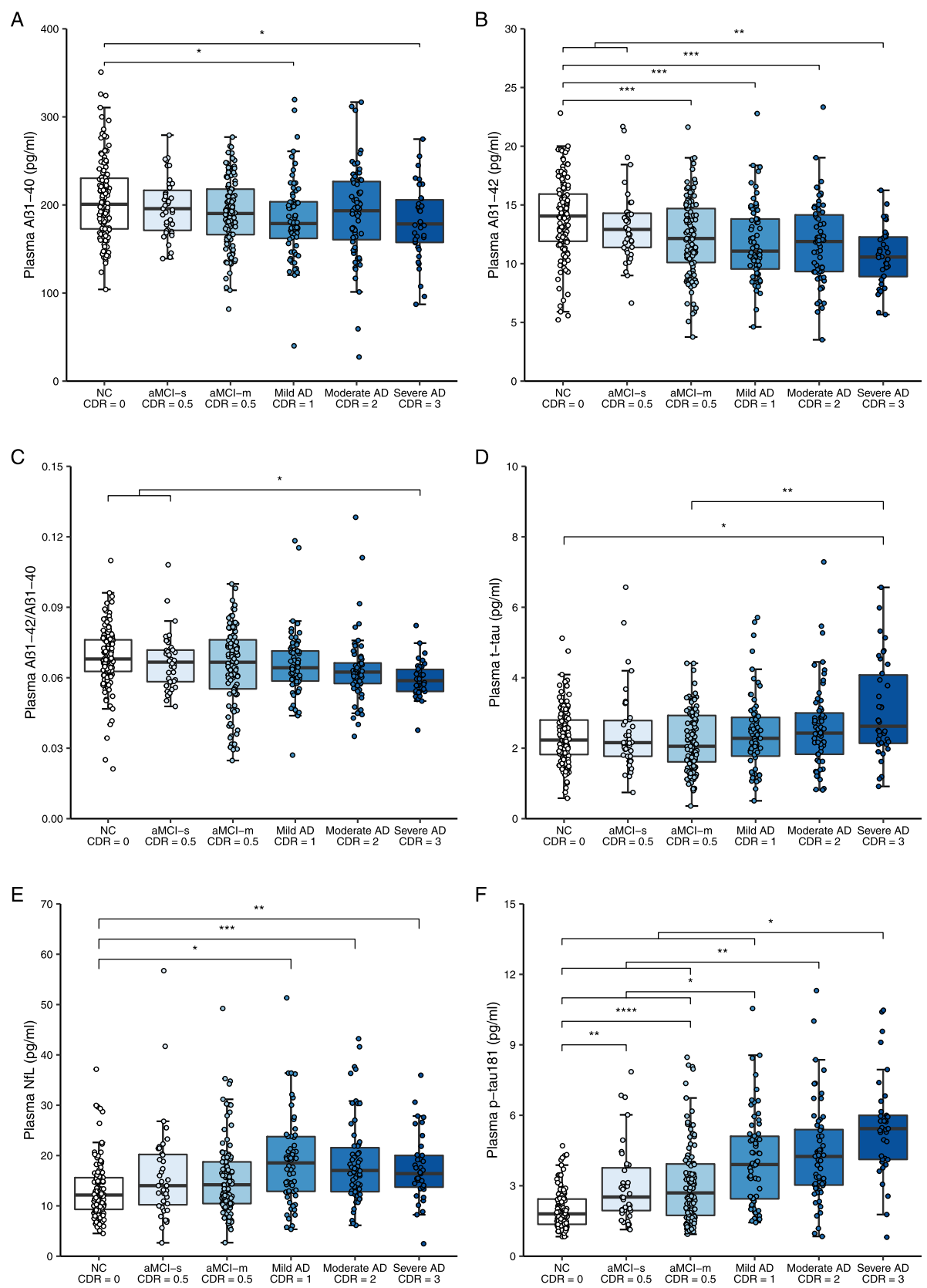

Fig. 1 Plasma biomarkers in participants with different clinical cognitive status. Note: The ANOVA and the post hoc Tukey test were used for comparison of plasma $A \beta_{1-40} \& A \beta_{1-42}$ in six groups, while Welch test and the post hoc Games-Howell test were used to compare the plasma $A \beta_{1-42} / A \beta_{1-40}$, t-tau, NfL, and p-tau181 among six groups. Six extreme values were not shown in panel E, but they were included in the statistical analyses. The multiple corrected $P$ values were presented with asterisks: ${ }^{*} P<0.05,{ }^{* *} P<0.01,{ }^{* * *} P<0.001$, and ${ }^{* * * *} P<0.0001$. NC, normal cognition; aMCl-s, amnestic mild cognitive impairment-single domain; aMCl-m, amnestic mild cognitive impairment-multiple domains; $A D$, Alzheimer's disease clinical syndrome; CDR, Clinical Dementia Rating Scale; A $\beta$, amyloid-beta protein; t-tau, total tau; NfL, neurofilament protein light chain; p-tau181, tau phosphorylated at threonine 181

$\geq 1$ after Bonferroni correction $(r=-0.301, \mathrm{df}=161, P<$ 0.001) (Supplementary Table 2).

\section{Discussion}

The present study demonstrated that plasma $A \beta_{1-40}$, $\mathrm{A} \beta_{1-42}$, and $A \beta_{1-42} / A \beta_{1-40}$ had a decreasing trend, while plasma t-tau, NfL, and p-tau181 escalated along the different stages of cognitive impairment as the CDR score increased from 0 to 3 . P-tau181 was the best plasma indicator of clinical cognitive performance and had correlations with broader cognitive domains than the other five biomarkers. To our knowledge, it was the first study 


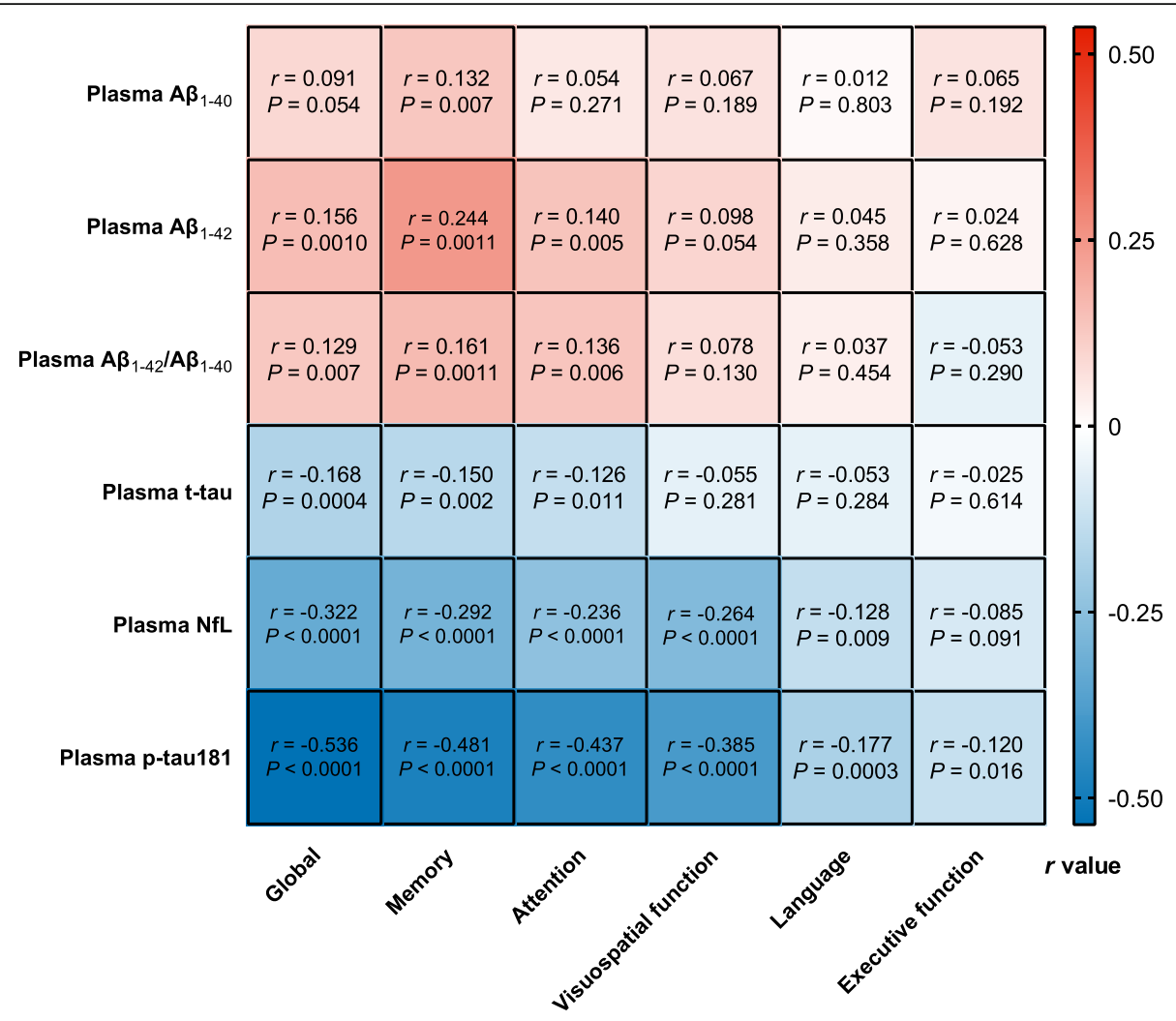

Fig. 2 Correlations between plasma biomarkers and cognitive domains. Note: The plasma biomarkers concentrations were log transformed. The partial correlation coefficients ( $r$ ) were adjusted for age, gender, and education year. $\mathrm{P}<0.0014$ was considered statistically significant after using multiple comparisons by Bonferroni correction. A $\beta$, amyloid-beta protein; $\mathrm{t}$-tau, total tau; NfL, neurofilament protein light chain; $\mathrm{p}$-tau181, tau phosphorylated at threonine 181

to exhibit the distribution of plasma p-Tau 181 using the Simoa HD-X platform in Chinese older individuals with different stages of $\mathrm{AD}$ clinical syndrome.

Pathology is pivotal in the diagnosis of AD. However, cognitive performance, including various cognitive domains, played a more significant role in patient management and efficacy evaluation. Cognitive manifestations are closely related to one's daily function and quality of life for both patients and caregivers. Thus, we focused on individual's performance and classified the participants into six groups according to CDR levels and clinical cognitive diagnosis. One previous study found plasma p-tau181 increased at preclinical $\mathrm{AD}$ and further increased at the MCI and dementia stages [3]. Another group verified that plasma $\mathrm{p}$-tau181 gradually increased from the $A \beta$-negative cognitively unimpaired older adults, through the $A \beta$-positive cognitively unimpaired and MCI groups, to the highest concentrations in $A \beta$ positive $\mathrm{MCI}$ and $\mathrm{AD}$ groups [4]. Our results testified their findings from the clinical perspective and further indicated that p-tau181 was a symptom-related plasma biomarker. Although some markers showed a significant escalating or descending trend along the AD spectrum, plasma p-tau181 is the only one that had a significant discrepancy in the later stage of $\mathrm{AD}$ clinical syndrome with overt dementia symptoms. This means plasma ptau181 may keep increasing along with the deterioration of cognitive function, till the severe stage of AD. Previous studies showed that plasma p-tau181 was correlated with CSF p-tau181 in AD patients, suggesting that plasma p-tau181 was originated from the central nervous system [3, 4]. Peripheral p-tau reflects the phosphorylation of the tau protein, which eventually leads to the neurofibrillary tangles in the brain [8]. Therefore, the continuous increment of the plasma p-tau across different stages of $\mathrm{AD}$ indicated the ongoing tau-related pathologic change in the brain, even in the moderate to severe stage of the disease. This may potentially guide the development of disease-modifying therapy for the advanced-stage AD patients in the future.

Previously, one cohort study demonstrated that plasma p-tau181 was correlated with the MMSE score [4]. However, the relationships between plasma p-tau181 and different cognitive domains have not been reported. Our study revealed a strong correlation between plasma ptau181 and various cognitive domains. The correlation between p-tau181 and MMSE was only observed in participants with dementia symptoms. This may reflect the 


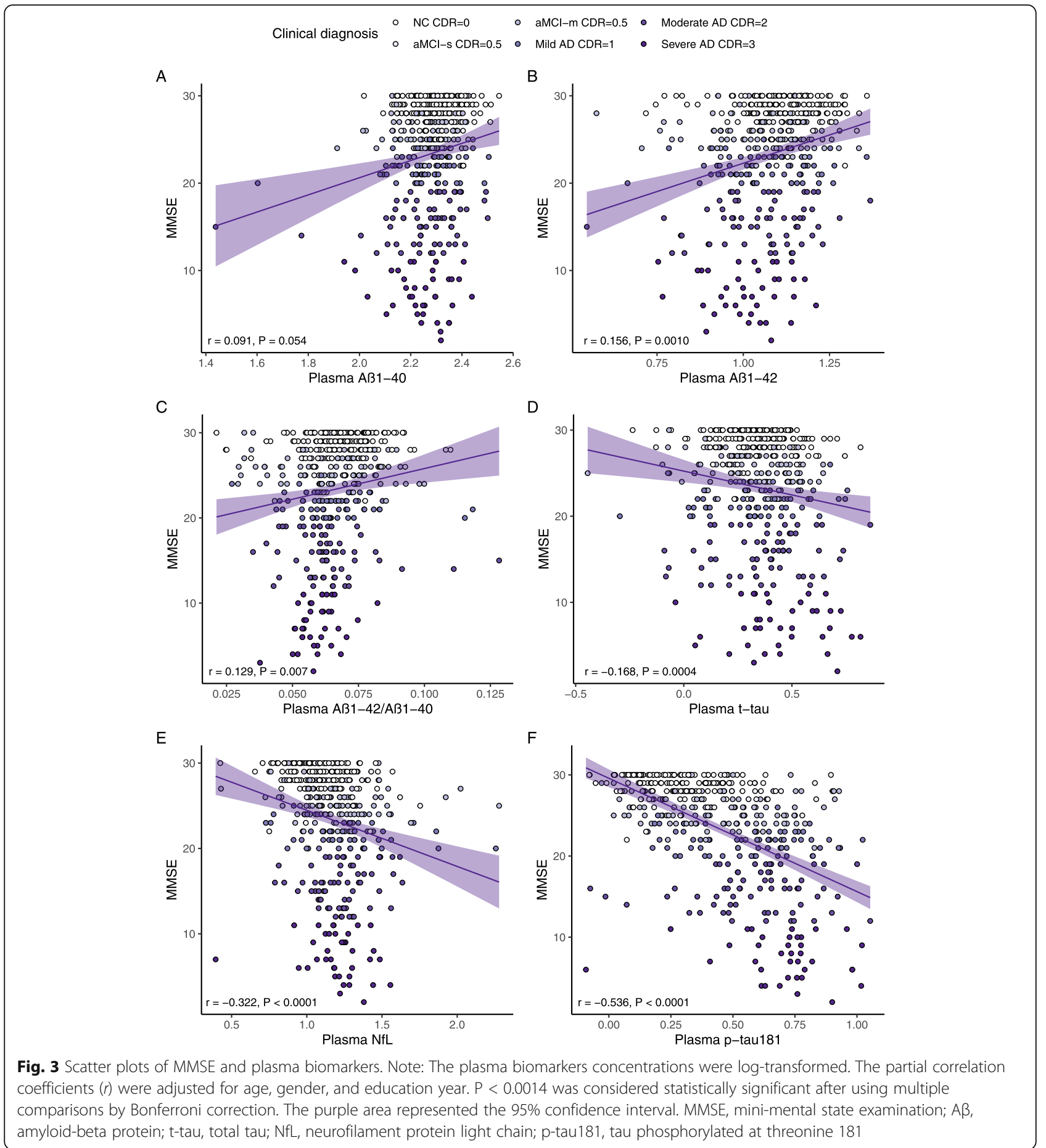

underlying mechanism that plasma p-tau181 is regulated differently by the disease staging, namely the Alzheimer's pathological status in the brain. Another possibility is that the narrow range of the testing scales, as well as the "ceiling effects" or "floor effects," might also weaken the correlation between plasma biomarkers and cognition. However, this might not be the main reason, since when analyzing with the more sensitive tests assessing memory and attention, the correlations were still absent in the subgroup with $\mathrm{CDR}=0$.

The NfL was an age-related biomarker [49]. Lin et al. found that higher plasma NfL levels correlated with lower MMSE scores [15], without adjustment for age. Another study by Chatterjee et. al. showed that plasma 


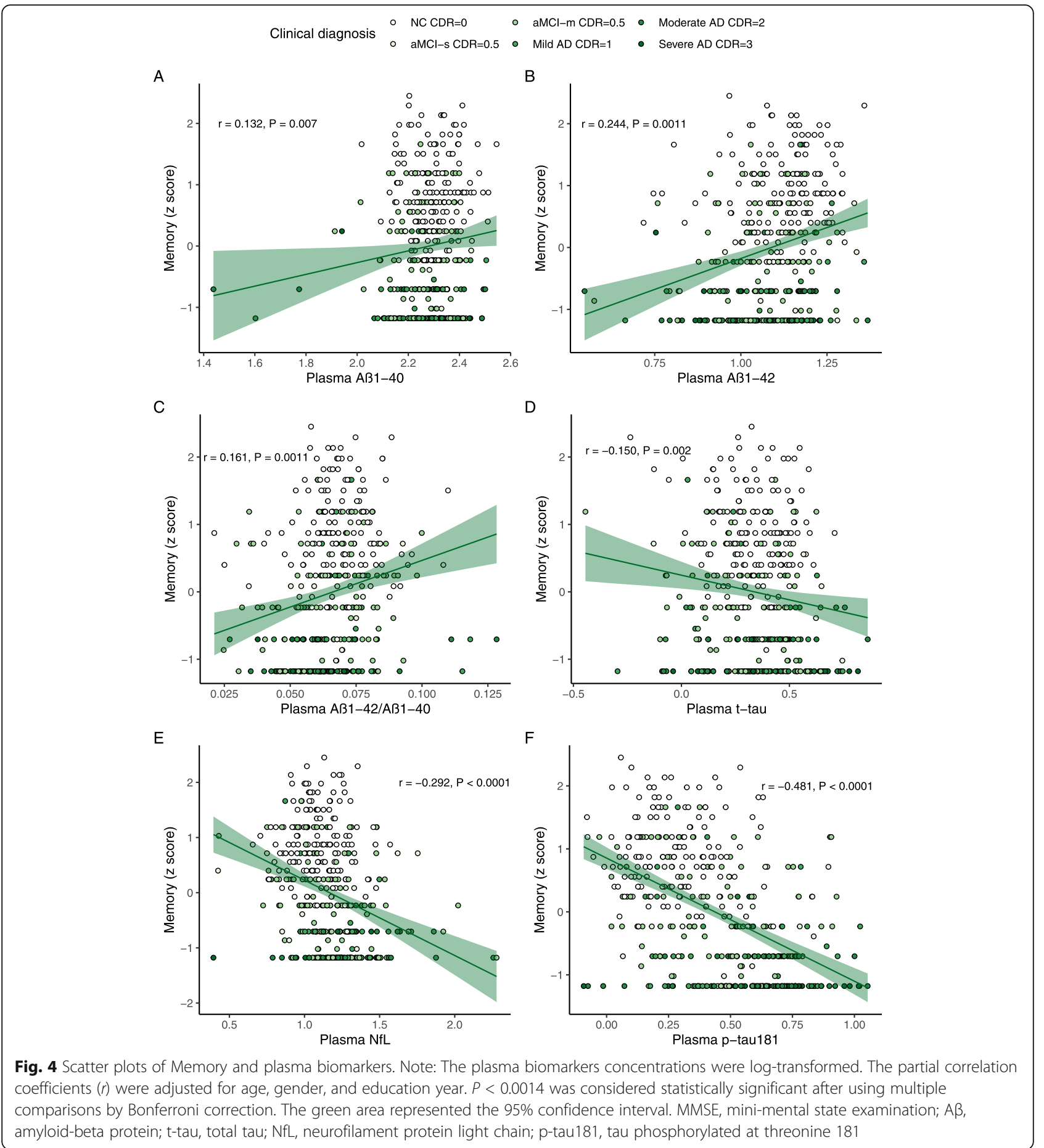

NfL was inversely correlated with working memory, executive function, and the global composite score after considering age [14]. Two other studies found plasma NfL associated with all cognitive domains after adjusting potential confounders including age [21, 22]. However, using the same Simoa detecting method, a Chinese group did not find any correlation of NfL with episodic memory, information processing speed, executive function, or visuospatial function after adjusting for age, gender, and education. In our results, the plasma NfL had a significant correlation after adjustment for age and other covariates, not only with global cognition, but also with the other three cognitive domains. This suggested that, although NfL was regarded as a non-specific biomarker 
for neurological diseases [12], it still has value in the monitoring of $\mathrm{AD}$ cognitive deterioration. Nevertheless, most correlations could not be observed when we classified participants into three sub-groups according to CDR levels of $0,0.5$, and $\geq 1$. The paradoxical results may be attributed to the diverse pathophysiological conditions in different cognitive stages [49].

The traditional amyloid cascade theory emphasized that $A \beta$ as an initial factor that triggers the following Tau pathology [1]. Our study found a relatively weaker correlation between the plasma amyloid biomarkers $\left(A \beta_{1-42}, A \beta_{1-42} / A \beta_{1-40}\right)$ and the cognition, only with MMSE and memory. Previous CSF and PET studies [50, 51] indicated that the amyloidrelated biomarkers reflected the earliest pathological change but tended to reach a plateau as the disease progressed to the dementia stage. Since the plasma $\mathrm{A} \beta$ was supposed to display the central nervous change [52], blood $A \beta$ level may also be saturated in the symptomatic individuals. However, in the CDR subgroups, the trend is ambiguous and inconsistent, probably because of the small sample and minor effect size. Further large-scale longitudinal studies need to be conducted to demonstrate the dynamic $A \beta$ alteration along the spectrum of $\mathrm{AD}$. A previous experimental study showed that neurons exposed to $\mathrm{A} \beta$ had increased synthesis and secretion of tau [53]. These neurons may eventually degenerate and develop tangle pathology, which may also drive the increase of p-tau in the blood. Therefore, regarding $A D$ as a lengthy, continuous disease course, plasma p-tau181, being more sensitive and clinical-relevant, might be superior to the amyloid-related markers.

\section{Limitations}

There are several limitations to our study. Firstly, the biomarkers in this study were only detected once without longitudinal measurements. However, we separated the participants into six groups according to CDR scores and cognitive diagnosis to simulate the spectrum of $\mathrm{AD}$ clinical syndrome. Future prospective studies are needed to verify our findings. Secondly, the participants in our studies were from two different resources with unavoidable imparity. However, there was no significant difference in some dementia-related risk factors, such as smoking, alcohol consumption, hypertension, and diabetes mellitus (Table 1). Age, gender, and education year were adjusted in the multivariate statistical models. Thirdly, some neuropsychological tests might have "ceiling effects" (e.g., Go/No-Go Task) or "floor effects" (e.g., Auditory Verbal Learning Test), which may conceal the relationship between the biomarkers and the cognitive domains. Tests assessing similar cognitive domains have unavoidable learning effects $[54,55]$ and intrusion errors
[56], which may lead to the unstable evaluation of the cognitive performance of the study participants. In our study, $38.6 \%$ of the intrusion rate of the memory tests (Supplementary Table 3) may impact the correlation estimations between the biomarkers and the cognitive domains. Fourthly, not all the participants were able to accomplish the extensive testing. About $90 \%$ of the participants accomplished tests for domains of memory, executive function, attention, and language, while $86 \%$ accomplished tests for the domain of the visuospatial function. Particularly in participants with severe AD, the response rates were even lower, due to their lack of ability to accomplish all the tests. When we repeated the analyses in participants that were able to accomplish all the tests $(\mathrm{N}=374)$, the most correlations still hold, including associations between p-tau181, NfL and global cognition, memory, attention, and visuospatial function; between $\mathrm{t}$-tau and global cognition; and between $A \beta 42$, $\mathrm{A} \beta 42 / \mathrm{A} \beta 40$, and memory (Supplementary Table 4). Although the correlation between plasma A42 and global cognition, language and p-tau181 could not pass the Bonferroni correction, and the $P$ values were still $<0.01$. Considering the Bonferroni correction as a conservative test, the results and conclusions could still be seen as solid. Fifthly, since AD was regarded as a continuum, the stage-partitioned correlations should be interpreted with caution due to the restricted range of cognitive test scores within each partition of disease severity. Lastly, we diagnosed $\mathrm{AD}$ based on the clinical standard rather than pathological evidence of CSF or amyloid/tau PET. Lacking a golden standard impeded us from the classifying of "ATN" framework [57] or receiver operating characteristic analysis.

\section{Conclusions}

In conclusion, we found plasma p-tau181 increased in the AD clinical syndrome. Plasma p-tau181 had correlations with broader cognitive domains than other biomarkers. Our study suggests that the plasma p-tau181 may be a promising, clinical-relevant blood-based biomarker. Longitudinal studies are needed to verify these findings and provide more evidence for the association between plasma p-tau181 and cognitive manifestations.

\footnotetext{
Abbreviations

AD: Alzheimer's disease; aMCl: Amnestic mild cognitive impairment; aMClm: Amnestic mild cognitive impairment multi-domains; aMCl-s: Amnestic mild cognitive impairment single-domain; ANOVA: Analysis of variance;

APOE: Apolipoprotein E; CDR: Clinical Dementia Rating; CSF: Cerebrospinal fluid; EDTA: Ethylene diamine tetraacetic acid; MCl: Mild cognitive impairment; MMSE: Mini-mental state examination; MoCA-B: Montreal Cognitive Assessment-Basic; NC: Normal cognition; NfL: Neurofilament protein light chain; PET: Positron emission tomography; p-tau181: Phosphorylated tau 181; SAS: Shanghai Aging Study; SD: Standard deviation; Simoa: Single-molecule array; TMT: Trail Making Test; t-tau: Total tau
} 


\section{Supplementary Information}

The online version contains supplementary material available at https://doi. org/10.1186/s13195-021-00864-x.

Additional file 1: Supplementary Table 1. Domain-specific cognition extracted from neuropsychological tests in the current study.

Additional file 2: Supplementary Table 2. Correlations between plasma biomarkers and global \& domain-specific cognition in participants with different $A D$ stages

Additional file 3: Supplementary Table 3. Intrusion of the Memory tests. Additional file 4: Supplementary Table 4. Correlations between plasma biomarkers and cognitive domains in participants that were able to accomplish all tests $(N=374)$.

\section{Acknowledgements}

Not applicable

\section{Authors' contributions}

QZ, HX, and DD developed the original idea. ZX and XW searched the literature. ZX, WW, XL, SD, and LZ collected the samples and data. XW and JY measured the blood biomarkers with support from HG. ZX and JL analyzed the data. ZX and XW wrote the manuscript. QZ, HX, and DD revised the manuscript. The authors read and approved the final manuscript.

\section{Funding}

This work was supported by grants of the Clinical Research Plan of SHDC (No. SHDC2020CR4007), Key Projects of Special Development Funds for the Shanghai Zhangjiang National Innovation Demonstration Zone (201905-XHCHJ-H25-201), MOE Frontiers Center for Brain Science (JIH2642001/028), Scientific Research Plan Project of Shanghai Science and Technology Committee (17411950106, 17411950701), National Project of Chronic Disease (2016YFC1306402), National Natural Science Foundation of China (82071200, 81773513, 21874091, 31927803), and Shanghai Municipal Science and Technology Major Project (2018SHZDZX01) and ZJLab.

\section{Availability of data and materials}

The datasets used and/or analyzed during the current study are available from the corresponding author on reasonable request.

\section{Ethics approval and consent to participate}

This study was approved by the Medical Ethics Committee of Huashan Hospital, Fudan University, Shanghai, China (No. 2009-195 and 2011-288). All participants and/or their legal proxy gave written informed consent to participate.

\section{Consent for publication}

Not applicable

\section{Competing interests}

The authors declare that they have no competing interests.

\section{Author details}

'Institute of Neurology, Huashan Hospital, Fudan University, 12 Wulumuq Zhong Rd, Shanghai, China. ${ }^{2}$ National Clinical Research Center for Aging and Medicine, Huashan Hospital, Fudan University, Shanghai, China. ${ }^{3}$ School of Biomedical Engineering, Shanghai Jiao Tong University, Shanghai, China. ${ }^{4}$ Department of Biostatistics, School of Public Health, Fudan University, Shanghai, China. ${ }^{5}$ Key Lab of Public Health Safety of the Ministry of Education, Fudan University, Shanghai, China.

Received: 5 February 2021 Accepted: 20 June 2021

Published online: 05 July 2021

\section{References}

1. Scheltens P, Blennow K, Breteler MMB, de Strooper B, Frisoni GB, Salloway S, et al. Alzheimer's disease. Lancet. 2016;388(10043):505-17.

2. Blennow K, Zetterberg H. Biomarkers for Alzheimer's disease: current status and prospects for the future. J Intern Med. 2018;284(6):643-63. https://doi. org/10.1111/joim.12816.
3. Janelidze S, Mattsson N, Palmqvist S, Smith R, Beach TG, Serrano GE, et al. Plasma P-tau181 in Alzheimer's disease: relationship to other biomarkers, differential diagnosis, neuropathology and longitudinal progression to Alzheimer's dementia. Nat Med. 2020;26(3):379-86. https://doi.org/10.1038/ s41591-020-0755-1.

4. Karikari TK, Pascoal TA, Ashton NJ, Janelidze S, Benedet AL, Rodriguez JL, et al. Blood phosphorylated tau 181 as a biomarker for Alzheimer's disease: a diagnostic performance and prediction modelling study using data from four prospective cohorts. Lancet Neurol. 2020;19(5):422-33. https://doi.org/1 0.1016/S1474-4422(20)30071-5.

5. Lantero Rodriguez J, Karikari TK, Suarez-Calvet M, Troakes C, King A, Emersic A, et al. Plasma p-tau181 accurately predicts Alzheimer's disease pathology at least 8 years prior to post-mortem and improves the clinical characterisation of cognitive decline. Acta Neuropathol. 2020;140(3):267-78. https://doi.org/10.1007/s00401-020-02195-x.

6. Cullen NC, Leuzy A, Palmqvist S, Janelidze S, Stomrud E, Pesini P, et al. Individualized prognosis of cognitive decline and dementia in mild cognitive impairment based on plasma biomarker combinations. Nat Aging. 2020;1(1):114-23.

7. Park JC, Han SH, Yi D, Byun MS, Lee JH, Jang S, et al. Plasma tau/amyloid-beta142 ratio predicts brain tau deposition and neurodegeneration in Alzheimer's disease. Brain. 2019;142(3):771-86. https://doi.org/10.1093/brain/awy347.

8. Mielke MM, Hagen CE, Xu J, Chai X, Vemuri P, Lowe VJ, et al. Plasma phospho-tau181 increases with Alzheimer's disease clinical severity and is associated with tau- and amyloid-positron emission tomography. Alzheimers Dement. 2018;14(8):989-97. https://doi.org/1 0.1016/j.jalz.2018.02.013.

9. Barthélemy NR, Horie K, Sato C, Bateman RJ. Blood plasma phosphorylatedtau isoforms track CNS change in Alzheimer's disease. J Exp Med. 2020; 217(11):e20200861. https://doi.org/10.1084/jem.20200861.

10. Albani D, Marizzoni M, Ferrari C, Fusco F, Boeri L, Raimondi I, et al. Plasma A 42 as a biomarker of prodromal Alzheimer's disease progression in patients with amnestic mild cognitive impairment: evidence from the PharmaCog/E-ADNI study. J Alzheimers Dis. 2019;69(1):37-48. https://doi. org/10.3233/JAD-180321.

11. Zhou W, Zhang J, Ye F, Xu G, Su H, Su Y, et al. Plasma neurofilament light chain levels in Alzheimer's disease. Neurosci Lett. 2017;650:60-4. https://doi. org/10.1016/j.neulet.2017.04.027.

12. Mattsson N, Andreasson U, Zetterberg H, Blennow K. Association of plasma neurofilament light with neurodegeneration in patients with Alzheimer disease. JAMA Neurol. 2017;74(5):557-66. https://doi.org/10.1001/jama neurol.2016.6117.

13. Karikari TK, Benedet AL, Ashton NJ, Lantero Rodriguez J, Snellman A, SuarezCalvet $\mathrm{M}$, et al. Diagnostic performance and prediction of clinical progression of plasma phospho-tau181 in the Alzheimer's disease neuroimaging initiative. Mol Psychiatry. 2021;26(2):429-42. https://doi.org/1 0.1038/s41380-020-00923-z.

14. Chatterjee P, Goozee K, Sohrabi HR, Shen K, Shah T, Asih PR, et al. Association of plasma neurofilament light chain with neocortical amyloid-beta load and cognitive performance in cognitively normal elderly participants. J Alzheimers Dis. 2018;63(2):479-87. https:/doi.org/10.3233/JAD-180025.

15. Lin YS, Lee WJ, Wang SJ, Fuh JL. Levels of plasma neurofilament light chain and cognitive function in patients with Alzheimer or Parkinson disease. Sci Rep. 2018;8(1):17368. https://doi.org/10.1038/s41598-018-35766-w.

16. Shi Y, Lu X, Zhang L, Shu H, Gu L, Wang Z, et al. Potential value of plasma amyloid-beta, total tau, and neurofilament light for identification of early Alzheimer's disease. ACS Chem Neurosci. 2019;10(8):3479-85. https://doi. org/10.1021/acschemneuro.9b00095.

17. Lee JJ, Choi Y, Chung S, Yoon DH, Choi SH, Kang SM, et al. Association of plasma oligomerized beta amyloid with neurocognitive battery using Korean version of consortium to establish a registry for Alzheimer's disease in health screening population. Diagnostics (Basel). 2020;10(4):237. https:// doi.org/10.3390/diagnostics10040237.

18. Lim YY, Maruff P, Kaneko N, Doecke J, Fowler C, Villemagne VL, et al. Plasma amyloid-beta biomarker associated with cognitive decline in preclinical Alzheimer's disease. J Alzheimers Dis. 2020;77(3):1057-65. https://doi.org/1 0.3233/JAD-200475.

19. Tsai CL, Liang CS, Lee JT, Su MW, Lin CC, Chu HT, et al. Associations between plasma biomarkers and cognition in patients with Alzheimer's disease and amnestic mild cognitive impairment: a cross-sectional and Iongitudinal study. J Clin Med. 2019;8(11):1893. https://doi.org/10.3390/ jcm8111893. 
20. Sugarman MA, Zetterberg H, Blennow K, Tripodis Y, McKee AC, Stein TD, et al. A longitudinal examination of plasma neurofilament light and total tau for the clinical detection and monitoring of Alzheimer's disease. Neurobiol Aging. 2020;94:60-70. https://doi.org/10.1016/j.neurobiolaging.2 020.05.011.

21. Verberk IMW, Thijssen E, Koelewijn J, Mauroo K, Vanbrabant J, de Wilde A, et al. Combination of plasma amyloid beta(1-42/1-40) and glial fibrillary acidic protein strongly associates with cerebral amyloid pathology. Alzheimers Res Ther. 2020;12(1):118. https://doi.org/10.1186/s13195-02000682-7.

22. Dage JL, Wennberg AMV, Airey DC, Hagen CE, Knopman DS, Machulda MM et al. Levels of tau protein in plasma are associated with neurodegeneration and cognitive function in a population-based elderly cohort. Alzheimers Dement. 2016;12(12):1226-34. https://doi.org/10.1016/j.jalz.2016.06.001.

23. Ding D, Zhao Q, Guo Q, Meng H, Wang B, Yu P, et al. The Shanghai Aging Study: study design, baseline characteristics, and prevalence of dementia. Neuroepidemiology. 2014;43(2):114-22. https://doi.org/10.1159/000366163.

24. Luo J, Zhu G, Zhao Q, Guo Q, Meng H, Hong Z, et al. Prevalence and risk factors of poor sleep quality among Chinese elderly in an urban community: results from the Shanghai aging study. PLoS One. 2013;8(11): e81261. https://doi.org/10.1371/journal.pone.0081261.

25. Mahoney FI, Barthel DW. Functional evaluation: the Barthel index. Md State Med J. 1965;14:61-5.

26. Lawton MP, Brody EM. Assessment of older people: self-maintaining and instrumental activities of daily living. Gerontologist. 1969;9(3):179-86. https://doi.org/10.1093/geront/9.3_Part_1.179.

27. Smirnov DA, Morley M, Shin E, Spielman RS, Cheung VG. Genetic analysis of radiation-induced changes in human gene expression. Nature. 2009; 459(7246):587-91. https://doi.org/10.1038/nature07940.

28. Zhang MY, Katzman R, Salmon D, Jin H, Cai GJ, Wang ZY, et al. The prevalence of dementia and Alzheimer's disease in Shanghai, China: impact of age, gender, and education. Ann Neurol. 1990;27(4):428-37. https://doi. org/10.1002/ana.410270412.

29. Chen $K L, X u Y, C h u A Q$, Ding D, Liang XN, Nasreddine $Z S$, et al. Validation of the Chinese version of montreal cognitive assessment basic for screening mild cognitive impairment. J Am Geriatr Soc. 2016;64(12):e285-e90. https:// doi.org/10.1111/jgs.14530.

30. Huang L, Chen KL, Lin BY, Tang L, Zhao QH, Lv YR, et al. Chinese version of Montreal Cognitive Assessment Basic for discrimination among different severities of Alzheimer's disease. Neuropsychiatr Dis Treat. 2018;14:2133-40. https://doi.org/10.2147/NDT.S174293.

31. Huang YY, Qian SX, Guan QB, Chen KL, Zhao QH, Lu JH, et al. Comparative study of two Chinese versions of Montreal Cognitive Assessment for Screening of Mild Cognitive Impairment. Appl Neuropsychol Adult. 2021; 28(1):88-93. https://doi.org/10.1080/23279095.2019.1602530.

32. Ding D, Zhao Q, Guo Q, Meng H, Wang B, Luo J, et al. Prevalence of mild cognitive impairment in an urban community in China: a cross-sectional analysis of the Shanghai Aging Study. Alzheimers Dement. 2015;11(3):300-9 e2. https://doi.org/10.1016/j.jalz.2013.11.002.

33. Zhao Q, LV Y, Zhou Y, Hong Z, Guo Q. Short-term delayed recall of auditory verbal learning test is equivalent to long-term delayed recall for identifying amnestic mild cognitive impairment. PLoS One. 2012;7(12):e51157. https:// doi.org/10.1371/journal.pone.0051157.

34. Guo Q, Zhao Q, Chen M, Ding D, Hong Z. A comparison study of mild cognitive impairment with 3 memory tests among Chinese individuals. Alzheimer Dis Assoc Disord. 2009;23(3):253-9. https://doi.org/10.1097/WAD. Ob013e3181999e92.

35. Zhou B, Zhao Q, Kojima S, Ding D, Higashide S, Nagai Y, et al. One-year outcome of Shanghai mild cognitive impairment cohort study. Curr Alzheimer Res. 2019;16(2):156-65. https://doi.org/10.2174/15672050166661 81128151144

36. Weixiong S, Chuanzhen LV, Yimin S, LV CZ, Guo QH. Boston naming test in Chinese elderly: patient with mild cognitive impairment and Alzheimer's dementia. Chin Ment Health J. 2006;20:81-4.

37. Lu J, Guo Q, Hong Z, Shi W, Lu C. Trail making test used by Chinese elderly patients with mild cognitive impairment and mild Alzheimer's dementia. Chinese J Clin Psychol. 2006;14:118-21.

38. Nasreddine ZS, Phillips NA, Bédirian V, Charbonneau S, Whitehead V, Collin I, et al. The Montreal Cognitive Assessment, MoCA: a brief screening tool for mild cognitive impairment. J Am Geriatr Soc. 2005;53(4):695-9. https://doi. org/10.1111/j.1532-5415.2005.53221.x.
39. Morris JC. The Clinical Dementia Rating (CDR): current version and scoring rules. Neurology. 1993;43(11):2412-4. https://doi.org/10.1212/ wnl.43.11.2412-a.

40. Lim WS, Chong MS, Sahadevan S. Utility of the clinical dementia rating in Asian populations. Clin Med Res. 2007;5(1):61-70. https://doi.org/10.3121/ cmr.2007.693.

41. American Psychiatric Association: diagnostic and statistical manual of mental disorders, ed 4. Washington, American Psychiatric Association, 1994, p. 143-147.

42. McKhann G, Drachman D, Folstein M, Katzman R, Price D, Stadlan EM. Clinical diagnosis of Alzheimer's disease: report of the NINCDS-ADRDA Work Group under the auspices of Department of Health and Human Services Task Force on Alzheimer's Disease. Neurology. 1984;34(7):939-44. https://doi org/10.1212/WNL.34.7.939.

43. Petersen RC. Mild cognitive impairment as a diagnostic entity. J Intern Med. 2004;256(3):183-94. https://doi.org/10.1111/j.1365-2796.2004.01388.x.

44. Petersen RC. Clinical practice. Mild cognitive impairment. New Engl J Med. 2011;364(23):2227-34. https://doi.org/10.1056/NEJMcp0910237.

45. Petersen RC, Thomas RG, Grundman M, Bennett D, Doody R, Ferris S, et al. Vitamin $\mathrm{E}$ and donepezil for the treatment of mild cognitive impairment. New Engl J Med. 2005;352(23):2379-88. https://doi.org/10.1 056/NEJMoa050151.

46. Halawa OA, Gatchel JR, Amariglio RE, Rentz DM, Sperling RA, Johnson KA, et al. Alzheimer's disease neuroimaging initiative. Inferior and medial temporal tau and cortical amyloid are associated with daily functional impairment in Alzheimer's disease. Alzheimers Res Ther. 2019;11(1):14

47. IBM Corp. Released 2017. IBM SPSS Statistics forWindows, Version 25.0. Armonk, NY: IBM Corp.

48. R Core Team. R: A language and environmentfor statistical computing. Vienna, Austria: R Foundation for Statistical Computing; 2020. https://www. R-project.org/

49. Gaetani L, Blennow K, Calabresi P, Di Filippo M, Parnetti L, Zetterberg H. Neurofilament light chain as a biomarker in neurological disorders. J Neurol Neurosurg Psychiatry. 2019;90(8):870-81. https://doi.org/10.1136/jnnp-201 8-320106.

50. Villemagne $V L$, Burnham S, Bourgeat P, Brown B, Ellis KA, Salvado O, et al. Amyloid $\beta$ deposition, neurodegeneration, and cognitive decline in sporadic Alzheimer's disease: a prospective cohort study. Lancet Neurol. 2013;12(4):357-67. https://doi.org/10.1016/S1474-4422(13)70044-9.

51. Haldenwanger A, Eling P, Kastrup A, Hildebrandt H. Correlation between cognitive impairment and CSF biomarkers in amnesic $\mathrm{MCl}$, non-amnesic MCl, and Alzheimer's disease. J Alzheimers Dis. 2010;22(3):971-80. https:// doi.org/10.3233/JAD-2010-101203.

52. Ovod V, Ramsey KN, Mawuenyega KG, Bollinger JG, Hicks T, Schneider T, et al. Amyloid beta concentrations and stable isotope labeling kinetics of human plasma specific to central nervous system amyloidosis. Alzheimers Dement. 2017;13(8):841-9. https://doi.org/10.1016/j.jalz.2017.06.2266.

53. Busche MA, Hyman BT. Synergy between amyloid-beta and tau in Alzheimer's disease. Nat Neurosci. 2020;23(10):1183-93. https://doi.org/10.1 038/s41593-020-0687-6.

54. Weuve J, Proust-Lima C, Power MC, Gross AL, Hofer SM, Thiébaut R, et al. Guidelines for reporting methodological challenges and evaluating potential bias in dementia research. Alzheimers Dement. 2015;11(9):1098109. https://doi.org/10.1016/j.jalz.2015.06.1885.

55. Scharfen J, Jansen $\mathrm{K}$, Holling H. Retest effects in working memory capacity tests: a meta-analysis. Psychon Bull Rev. 2018;25(6):2175-99. https://doi.org/1 0.3758/s13423-018-1461-6.

56. De Anna F, Attali E, Freynet L, Foubert L, Laurent A, Dubois B, et al. Intrusions in story recall: when over-learned information interferes with episodic memory recall. Evidence from Alzheimer's disease. Cortex. 2008; 44(3):305-11. https://doi.org/10.1016/j.cortex.2006.08.001.

57. Jack CR Jr, Bennett DA, Blennow K, Carrillo MC, Dunn B, Haeberlein SB, et al. NIA-AA Research Framework: toward a biological definition of Alzheimer's disease. Alzheimers Dement. 2018;14(4):535-62. https://doi.org/10.1016/j.ja |z.2018.02.018

\section{Publisher's Note}

Springer Nature remains neutral with regard to jurisdictional claims in published maps and institutional affiliations. 